\title{
Oral Stereognosis in Children With Cleft Palate and Normal Children
}

\author{
Jalal Bakhtiyari ${ }^{1}$; Hooshang Dadgar ${ }^{2 *}$; Ali Sadolahi $^{1}$; Ahmad Reza Khatoonabadi ${ }^{2}$ \\ ${ }^{1}$ Speech Therapy Department, Faculty of Rehabilitation, Semnan University of Medical Sciences, Semnan, IR Iran \\ ${ }^{2}$ Speech Therapy Department, Faculty of Rehabilitation, Tehran University of Medical Sciences, Tehran, IR Iran \\ ${ }^{*}$ Corresponding author: Hooshang Dadgar, Speech Therapy Department, Faculty of Rehabilitation, Tehran University of Medical Sciences, Tehran, IR Iran. Tel: +98-2177533939, Fax: \\ +98-217636042, E-mail: hdadgar@sina.tums.ac.ir
}

Received: January 25, 2014; Revised: March 30, 2014; Accepted: May 5, 2014

\begin{abstract}
Background: There is an assumption that oral senses have important roles in development and production of speech sounds.
Objectives:The aim of this study was comparison of oral stereognosis ability and response time in two groups of children with cleft palate as well as normal children.

Patients and Methods: The study population comprised 20 children with cleft palate, six to nine years old, after first surgery, with normal intelligence, and 40 normal children with normal IQ levels and no speech-language disorders. In this cross-sectional study, oral stereognosis was investigated by identification of objects with different shapes and surface alterations, when placed in the mouth and without visual aid. Thirteen pieces of $5 \times 5 \times 1 \mathrm{~mm}$ dimensions were used, affixed by dental floss to prevent swallowing.

Results: There was a significant difference between the oral stereognosis and response time (recognition time) of children with cleft palate and that of normal children $(\mathrm{P}<0.05)$.

Conclusions: Cleft of the palate area can significantly decrease the intraoral sensations, resulting in elongation of the diagnosis time.
\end{abstract}

Keywords: Oral Stereognosis; Cleft Palate; Speech

\section{Background}

Sensory systems and sensory control are important in voluntary motor patterns involved in speech (1). Sensory feedbacks such as auditory and kinesthetic are necessary for normal development of speech $(2,3)$. Oral sensorimotor function has an important role in speech (4). Stereognosis is the ability to perceive the form of an object by using the sense of touch (5) and oral stereognosis is the ability to recognize the form or shape of objects placed in the oral cavity without use of vision $(6,7)$. It is the most highly developed and complex sensory function which is conveyed by trigeminal nerve and involves sensory information from mucosal receptors, specifically tongue, and receptors in the palate (8-11). Accurate sensory input and normal integrated motor activity are essential for normal oral functions such as speech $(7,12)$; thus, poor oral stereognosis can affect the oral motor behavior, which is involved in articulation of speech sounds $(9,12)$. Oral discrimination test can be used for evaluation of oral perceptual abilities. This assessment can be beneficial in planning and evaluation of the therapeutic effect. Knowledge of kinesthetic feedback in speech expression could affect the speech production and perception (13). Several researches have indicated that oral stereognosis ability is relevant to speech production. Andrews reported that children with cleft palate and articulation disorders have more errors in oral discrimination (14). It's noted that ability of two-point discrimination was poor in children with misarticulation of $/ \mathrm{r} /$ (15). In this function (oral ste- reognosis), anatomical areas such as lingual-palatal ones are involved $(8,16)$. General or local pathology in the palate, i.e. cleft palate, can affect the oral stereognosis. Although the contribution of palatal sensitivity in development of speech is not well recognized, it is assumed that it has a role in acquiring normal articulation (17).

\section{Objectives}

The purpose of this study was to compare the level of oral stereognosis in children with cleft palate and normal children, six to nine years old. It was hypothesized that oral stereognostic ability would be poorer in patients with cleft palate because of their pathological condition.

\section{Patients and Methods}

\subsection{Participants}

In this cross-sectional study, oral stereognosis was investigated in 20 children with cleft palate, six to nine years old, who had passed the first operation, with normal intelligence. These children were selected from speech therapy clinics heterogeneous in type of cleft palate (unilateral). The inclusion criteria were: 1) Unilatreral cleft palate without cleft lip 2) Children who did not have orofacial syndromes 3) Lack of speech and oral apraxia (use of informal test) 4) The ones who had passed the first operation. The control group consisted of 40 normal children (the same 


\section{Bakhtiyari J et al.}

age) with normal IQ levels and no speech-language disorders, randomly selected from schools in Tehran, Iran. The aim of oral stereognosis was recognition of shape and its time. Parents of all children (cleft palate and control groups) signed written informed consents. The children's IQ levels were determined through Wechsler test by a clinical psychologist.

\subsection{Procedure}

From the first oral stereognosis test designed by Grossman (14) to date, this ability has been tested in different ways, but has not been significantly modified. In this study, stereognosis investigation was made according to (15). The test consisted of identification of objects with different shapes and surface alterations, when placed in mouth and without visual aid. Thirteen pieces were made of acrylic self-cured with dimensions of $5 \times 5 \times 1 \mathrm{~mm}$ and affixed with dental floss to prevent swallowing (Figure 1). Three of these pieces were used in different orders for some participants. The test was carried out in a quiet environment and by one examiner. Pieces with different shapes were put in the oral cavity. Subjects were asked to use their tongue and palate to identify the shape and avoid biting the test pieces. The 10 presentation pieces were put in random order. Children had to point out the corresponding shape on the chart. Correct and incorrect responses as well as the time of identification were recorded. Dichotomous scoring was used; each child's response was scored as 1 for correct response or 0 for incorrect response. The time taken to identify the object was recorded using a chronometer. To ensure of accurate response, each of the 10 pieces was presented twice randomly and the score of each piece was recorded after the second presentation.

\subsection{Data Analysis}

Independent t-test was used to examine the differences between the two groups (cleft palate and normal children). A probability of less than 0.05 was accepted as significant. Data analysis was done using SPSS version 11.5 for windows.

\section{Results}

The studied group consisted of 20 children with cleft palates and 40 normal children. The age characteristics of the two groups are shown in Table 1 . There was no difference between the ages of the two groups $(\mathrm{P}=0.519)$. Table 1 shows oral stereognosis ability scores between cleft palate and normal children. The oral stereognosis ability score in normal subjects ( mean $=7.50, \mathrm{SD}=1.80$ ) was significantly higher than the children with cleft palate (mean $=4.1, \mathrm{SD}=$ 1.85). However, significant difference was found in the oral stereognosis ability score between cleft palate and normal children. Statistical analysis showed significant differences in the oral stereognosis ability response time between the two groups. The oral stereognosis ability response time (seconds) in normal children $($ mean $=2.02, \mathrm{SD}=0.14$ ) was significantly shorter compared with those of children with cleft palate $($ mean $=2.21, \mathrm{SD}=0.21)($ Table 1$)$.

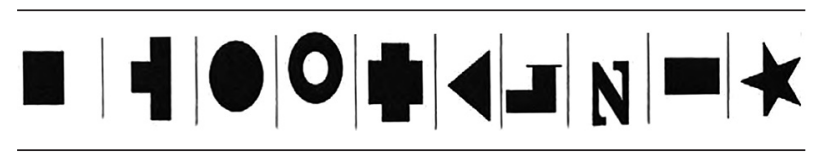

Figure 1. Oral Stereognostic Ability Test Pieces

Table 1. Characteristics and Response Times of Oral Stereognostic Scores Between the Two Groups ${ }^{\text {a }}$

\begin{tabular}{lccc}
\hline Parameter & $\begin{array}{c}\text { Cleft Palate } \\
\text { Subjects }(\mathbf{n}=\mathbf{2 0})\end{array}$ & $\begin{array}{c}\text { Normal } \\
\text { Subjects }(\mathbf{n}=\mathbf{4 0})\end{array}$ & PValue \\
\hline Age, $\mathbf{y}$ & $7.25 \pm 1.12$ & $7.45 \pm 1.13$ & 0.519 \\
$\begin{array}{l}\text { Oral stereognosis } \\
\text { score }\end{array}$ & $4.10 \pm 1.85$ & $7.50 \pm 1.80$ & 0.001 \\
Response time & $2.21 \pm 0.21$ & $2.02 \pm 0.13$ & 0.046
\end{tabular}

a Data are presented as Mean \pm SD.

\section{Discussion}

The purpose of our study was to evaluate the oral stereognosis ability in individuals with cleft palate and normal children six to nine years old. The results can be used to better understand the status of oral stereognosis in this population and develop intervention programs for children with cleft palate in the early years of their lives. Oral stereognosis ability has been studied in several researches to evaluate the oral perception $(6,7,14-16)$. This test is designed to evaluate the overall sensory and oral motor abilities. Oral stereognosis assessment may help the speech pathologist in planning treatment programs for children with oral dysfunctions. In various researches, different methods have been used for assessment of oral sensitivity, such as interdental size, oral form recognition, and two-point discrimination. In this research oral form of cognition and time of response was studied (7). The present study outcomes demonstrated that oral stereognosis ability was low in children with cleft palate in comparison with normal children. In spite of the differences in form and size of the pieces used for oral sensitivity assessment, results of our study were in agreement with those of previous researches. Engelen reported that combination of sensory inputs from the tongue and palate had a core role in oral perception of size (18). Hochberg and et al. studied oral stereognosis in patients with cleft palate, and reported that their stereognostic ability was inferior to that of normal subjects $(19,20)$. Andrews previously noted that subjects with cleft palate had poorer performances compared with healthy subject in oral form discrimination (21). We expected lower oral stereognosis scores for patients with cleft palate, simply because their palate conditions reduced perception of the oral cavity. Another more probable reason may have been the articulation disorders and reduction of speech movement in oral cavity, 
which may affect the sensory input coming from palate mechanoreceptors, not strong enough to confer higher stereognostic ability. The second reason was in agreement with previous researches $(19,21)$. Regarding the time of oral cognition, Uchiyama et al. reported that individuals with cleft palate had delay in the response time (22). In the patients studied, oral sensory function of the palate was significantly decreased. Surgery procedures entail manipulation of the palate to correct craniofacial deformities in children with cleft palate, which can affect craniofacial neurosensation including oral stereognosis ability. Evidence indicates that oral stereognosis has a significant role in speech. Hence, it seems necessary to evaluate and reinforce the intraoral sensation of these children in speech therapy programs.

\section{Acknowledgements}

We would like to thank all children who participated in this research and their parents.

\section{Authors' Contributions}

Hooshang Dadgar: write the article, Jalal Bakhtiyari: study design, data collection, Ahmad Reza Khatoonabadi: write the article, Ali Sadollahi: data collection.

\section{References}

1. Jones GB, Harris S, OliverW. Oral feedback: Variability of the effect on neuroblock anesthesia upon speech. J phon.1973;1:289-95.

2. Borden GJ. An interpretation of research of feedback interruption in speech. Brain Lang. 1979;7(3):307-19.

3. Attanasio JS. Relationships between oral sensory feedback skills and adaptation to delayed auditory feedback. J Commun Disord. 1987;20(5):391-402.

4. McNeil M. Clinical management of sensorimotor speech disorders. New York: Thieme; 2008.

5. Leung KC, Pow EH, McMillan AS, Wong MC, Li LS, Ho SL. Oral perception and oral motor ability in edentulous patients with stroke and Parkinson's disease. J Oral Rehabil. 2002;29(6):497-503.
6. Pow EH, Leung KC, McMillan AS, Wong MC, Li LS, Ho SL. Oral stereognosis in stroke and Parkinson's disease: a comparison of partially dentate and edentulous individuals. Clin Oral Investig. 2001;5(2):112-7.

7. Ahmed B, Hussain M, Yazdanie N. Oral stereognostic ability: a test of oral perception. J Coll Physicians Surg Pak. 2006;16(12):794-8.

8. Kawagishi S, Kou F, Yoshino K, Tanaka T, Masumi S. Decrease in stereognostic ability of the tongue with age. J Oral Rehabil. 2009;36(12):872-9.

9. Bhandari A, Hegde C, Prasad D. Relation between oral stereognosis and masticatory efficiency in complete denture wearers: an in vivo study. Braz J Oral Sci. 2010;9(3):358-61.

10. Patel JR. Comparative evaluation of effect of complete dentures on oral stereognosis in completely edentulous patients. Int J Clin Dent Sci. 2011;1(1).

11. Jacobs R, Bou Serhal C, van Steenberghe D. Oral stereognosis: a review of the literature. Clin Oral Investig. 1998;2(1):3-10.

12. Speirs RL, Maktabi MA. Tongue skills and clearance of toffee in two age-groups and in children with problems of speech articulation. ASDC J Dent Child.1990;57(5):356-60.

13. Bishop ME, Ringel RL, House AS. Orosensory perception, speech production, and deafness. J Speech Hear Res. 1973;16(2):257-66.

14. Andrews JR. Oral form discrimination in individuals with normal and cleft palates. Cleft Palate J.1973;10:92-8.

15. Scott CM, Ringel RL. Articulation without oral sensory control. J Speech Hear Res.1971;14(4):804-18.

16. do Vale Pedreira APR, Bonachela WC, Rossetti PHO, Pinto JHN. Evaluation of Oral Stereognosis in Dentate and Edentulous Subjects with and without Cleft Lip and Palate Before and After Chewing. Int J Oral Med Sci. 2004;3(1):61-5.

17. Noguchi M, Suda Y, Ito S, Kohama GI. Comparison of palatal sensitivity after treatment of cleft palate by a supraperiosteal or mucoperiosteal flap. Br J Oral Maxillofac Surg. 2004;42(5):432-5.

18. Engelen L, van der Bilt A, Bosman F. Relationship between oral sensitivity and masticatory performance. J Dent Res. 2004;83(5):388-92.

19. Hochberg I, Kabcenell J. Oral stereognosis in normal and cleft palate individuals. Cleft Palate J.1967;4:47-57.

20. Derakhshandeh F, Kolvani S, Najarzadeh F, Gheyasvand L, Falahati R. [The study and comparison of the mean of stereognosis in cleft palate and normal children]. J Rehabil. 2003;12:28-33.

21. Grossman R. Methods of determining oral tactile experience. In: Bosma JF editor. Second symposium on oral sensation and perception. 12th ed. Springfield: Charles C Thomas Publisher;1967. p. 161-181.

22. Uchiyama T, Nakano Y, Koeda H. Measurement of palatal surface sensation by neuro-sensory tests in the postoperative cleft palate patients. Bull Tokyo Dent Coll. 1998;39(4):243-9. 